Wilfrid Laurier University

Scholars Commons @ Laurier

Physics and Computer Science Faculty

Publications

Physics and Computer Science

1996

\title{
Phase Locking Between Fiske and Flux-Flow Modes in Coupled Sine-Gordon Systems
}

Niels Grønbech-Jensen

Los Alamos National Laboratory

James A. Blackburn

Wilfrid Laurier University, jabjabjab@cogeco.ca

Mogens R. Samuelsen

Technical University of Denmark

Follow this and additional works at: https://scholars.wlu.ca/phys_faculty

\section{Recommended Citation}

Grønbech-Jensen, Niels; Blackburn, James A.; and Samuelsen, Mogens R., "Phase Locking Between Fiske and Flux-Flow Modes in Coupled Sine-Gordon Systems" (1996). Physics and Computer Science Faculty Publications. 36.

https://scholars.wlu.ca/phys_faculty/36

This Article is brought to you for free and open access by the Physics and Computer Science at Scholars Commons @ Laurier. It has been accepted for inclusion in Physics and Computer Science Faculty Publications by an authorized administrator of Scholars Commons @ Laurier. For more information, please contact scholarscommons@wlu.ca. 


\title{
Phase locking between Fiske and flux-flow modes in coupled sine-Gordon systems
}

\author{
Niels Grønbech-Jensen \\ Theoretical Division, Los Alamos National Laboratory, Los Alamos, New Mexico 87545 \\ James A. Blackburn \\ Department of Physics and Computing, Wilfrid Laurier University, Waterloo, Ontario, Canada N2L $3 C 5$ \\ Mogens R. Samuelsen \\ Physics Department, The Technical University of Denmark, DK-2800 Lyngby, Denmark
}

(Received 7 December 1995)

\begin{abstract}
We investigate nonlinear resonant modes in coupled sine-Gordon systems with open boundary conditions. The system models coupled Josephson junctions with boundary conditions representing the situation where an external magnetic field is applied. The so-called Fiske modes are found to exist in phase-locked states where the equivalent voltages across the individual coupled Josephson junctions are either identical or identical with opposite signs. The analysis covers all Fiske modes including the flux-flow region. We present a comprehensive comparison between results on analytical treatment and direct numerical simulations of the coupled field equations. [S0163-1829(96)05718-9]
\end{abstract}

\section{INTRODUCTION}

Inductively coupled pairs of long Josephson junctions have been extensively investigated based on the experimental geometries and coupling mechanisms suggested in Refs. 1-6. The coupled system exhibits two different characteristic velocities for the linear ${ }^{1}$ and the nonlinear (relativistic) ${ }^{7,8}$ modes, and this feature has led to several interesting observations such as phase locking between various modes in the coupled systems, ${ }^{4,73}$ power emissions exceeding the superradiant limit, ${ }^{10,14,15}$ and stability of energetically unfavorable bound states of fluxon modes. ${ }^{9,16}$ Most theoretical analyses based on coupled and perturbed sine-Gordon systems have been done for the single soliton (fluxon) solutions, representing the zero-field dynamics of a trapped magnetic flux quantum in each junction. However, other interesting modes are relevant for this system. In particular, the so-called Fiske and flux-flow modes, ${ }^{17-24}$ arising when the system is placed in a magnetic field which is in the plane of the barrier and perpendicular to the long axis of the junction, are extremely stable and therefore useful in experimental configurations. ${ }^{10-12,25-30}$ These modes are characterized by resonances at the linear cavity frequencies and appear when the junctions are embedded in an external magnetic field. A subset of the Fiske resonances are called flux flow when the normalized frequency (voltage) is near the normalized external magnetic field (see below). We note that previous theoretical work has been published on flux-flow configurations in coupled systems ${ }^{31}$ as well as in single junctions, applying a moving kink soliton train solution as basis for the analysis. However, such an ansatz gives rise to a single resonance (the asymptotic propagation velocity) in the current-voltage characteristics and it can therefore not adequately describe the Fiske resonances within the flux-flow regime. The difficulty originates near the boundaries of the system, where, e.g., the injection process of a fluxon into the system, while keeping the boundary magnetic field constant, is incommensurate with the kink soliton solutions. As a result, the important details of multiple Fiske resonances are not considered in those treatments.

In this paper we generalize the Kulik theory ${ }^{18-20}$ to include phase-locked states between two inductively and/or capacitively coupled systems with aiding or opposing bias. This method describes the nonlinear modes as small resonant perturbations to a linear background. The advantage of this approach is that all the Fiske (cavity) resonances are represented-including the flux flow regime-as results of the damping, bias, and boundary conditions, and we can therefore obtain a reasonably good understanding of the complicated dynamics of phase-locked excitations in coupled Josephson systems in external magnetic fields.

The reality of the fabrication process implies that any sample of Josephson junctions is characterized by a distribution of system parameters. As a consequence it is never assured that two devices will respond with, e.g., the same frequency to the same external perturbation. Even if the systems are coupled, certain conditions must be satisfied before some form of synchronization can be obtained. The aim of this paper is to investigate the question of when the experimentally relevant Fiske modes can be expected to synchronize in a pair of coupled Josephson junctions (sineGordon systems). For simplicity we have decided to represent any difference between the two systems as a difference between their bias conditions. Thus, the coupled systems are modeled as being identical except for their individual bias. We note that differences in other parameters can easily be included in the analysis, ${ }^{32}$ but we have here decided to simplify the expressions by limiting the parameter space. In Sec. II we develop the analysis which gives explicit predictions for the existence of phase-locked states between any two Fiske (or flux-flow) modes in the two systems. Section III includes comparisons between the predictions and numerical simulations of the coupled sine-Gordon model. Section IV discusses the results and concludes the paper. 


\section{PERTURBATION ANALYSIS}

We investigate the configuration of two parallel coupled long Josephson junctions, ${ }^{7}$

$$
\begin{aligned}
& \phi_{x x}-\phi_{t t}-\sin \phi=\alpha \phi_{t}-\eta_{1}-\Delta_{1} \psi_{x x}-\Delta_{2} \psi_{t t}, \\
& \psi_{x x}-\psi_{t t}-\sin \psi=\alpha \psi_{t}-\eta_{2}-\Delta_{1} \phi_{x x}-\Delta_{2} \phi_{t t},
\end{aligned}
$$

where the boundary conditions for a stacked configuration ${ }^{2}$ are given by $\widetilde{\Gamma}$,

$$
\begin{aligned}
& \phi_{x}(0)=\phi_{x}(L)=\left(1+\Delta_{1}\right)^{-1} \widetilde{\Gamma}=\Gamma, \\
& \psi_{x}(0)=\psi_{x}(L)=\left(1+\Delta_{1}\right)^{-1} \widetilde{\Gamma}=\Gamma .
\end{aligned}
$$

In this model $\phi$ and $\psi$ describe the phase differences of the macroscopic superconducting quantum-mechanical wave functions measured across each of the two junctions. The spatial coordinate, $x$, is normalized to the characteristic Josephson length, $\lambda_{J} \approx \sqrt{\hbar / 2 e d \mu_{0} I_{c}}$, and the normalized length of the systems is given by $L$. The temporal dimension, $t$, is normalized to the inverse Josephson plasma frequency, $\omega_{J}^{-1} \approx \sqrt{\hbar \epsilon / 2 e t_{o} I_{c}}$. Here, the permeability is given by $\mu_{0}$, the permittivity by $\epsilon$, and the critical current density is $I_{c}$. The electric and magnetic thicknesses of the junction are given by the insulating layer $t_{o}$ and $d=2 \lambda_{L}+t_{o}, \lambda_{L}$ being the magnetic penetration depth of the superconductors. Tunneling of super current is represented by the sine terms, quasiparticle tunneling is specified by the dissipation parameter $\alpha$, and the normalized bias currents forced through the junctions are $\eta_{1}$ and $\eta_{2}$, respectively. The coupling mechanisms are given by the parameters $\Delta_{1}$ (inductive) (i, $^{3,4}$ and $\Delta_{2}$ (capacitive). ${ }^{7}$ The normalized external magnetic field, which is in the plane of each junction and perpendicular to their long axes, is given by $\widetilde{\Gamma}$.

We note that for the "side by side" geometry of junctions described in Refs. 4 and 5 the boundary conditions are

$$
\begin{gathered}
\phi_{x}(0)=\phi_{x}(L)=\left(1-\Delta_{1}\right)^{-1} \widetilde{\Gamma}=\Gamma, \\
-\psi_{x}(0)=-\psi_{x}(L)=\left(1-\Delta_{1}\right)^{-1} \widetilde{\Gamma}=\Gamma .
\end{gathered}
$$

However, in the following we will only consider the boundary conditions given by Eqs. (3) and (4), since the analysis, numerical simulations, and results are qualitatively very similar to the treatment below.

In place of Eqs. (1)-(4) we can rewrite the dynamical equations in terms of the phase variables, $u$ and $v$, given by

$$
\begin{aligned}
& u=\frac{\phi+\psi}{2}, \\
& v=\frac{\phi-\psi}{2},
\end{aligned}
$$

in which case the dynamical equations are

$$
\begin{aligned}
& \left(1+\Delta_{1}\right) u_{x x}-\left(1-\Delta_{2}\right) u_{t t}-\sin u \cos v=\alpha u_{t}-\eta_{s}, \\
& \left(1-\Delta_{1}\right) v_{x x}-\left(1+\Delta_{2}\right) v_{t t}-\sin v \cos u=\alpha v_{t}-\eta_{d} .
\end{aligned}
$$

The new bias parameters are:

$$
\begin{aligned}
& \eta_{s}=\frac{\eta_{1}+\eta_{2}}{2}, \\
& \eta_{d}=\frac{\eta_{1}-\eta_{2}}{2},
\end{aligned}
$$

and the appropriate boundary conditions are given by

$$
\begin{gathered}
u_{x}(0)=u_{x}(L)=\left(1+\Delta_{1}\right)^{-1} \widetilde{\Gamma}=\Gamma, \\
v_{x}(0)=v_{x}(L)=0 .
\end{gathered}
$$

We will now focus our attention to phase-locked Fiske (and flux flow) solutions of equations (1) and (2). The following treatment is further limited to the cases where the phases $\phi$ and $\psi$ evolve according to

$$
\left|\left\langle\int_{0}^{L} \phi_{t} d x\right\rangle\right|=\left|\left\langle\int_{0}^{L} \psi_{t} d x\right\rangle\right|,
$$

where \langle\rangle indicates temporal averaging. This is the simplest of a number of possible definitions of locking, but other cases of phase-locked dynamics can be obtained using the same formalism as presented below. Following the approach in Refs. 18, 19, 33, and 34, where the Fiske modes in a single junction were treated, we apply trial functions for the coupled system of the form

$$
\begin{gathered}
\phi=\phi_{0}+\Gamma x+\omega t+\sum_{m=0}^{\infty}\left(A_{m} \cos \omega t+B_{m} \sin \omega t\right) \cos k_{m} x, \\
\psi=\psi_{0}+\Gamma x+\sigma \omega t+\sum_{m=0}^{\infty}\left(C_{m} \cos \omega t+D_{m} \sin \omega t\right) \cos k_{m} x,
\end{gathered}
$$

where $\phi_{0}$ and $\psi_{0}$ are constants, $k_{m}=m \pi / L$, and $\sigma= \pm 1$ determines the sign of the frequency (normalized voltage). These trial functions assume monochromatic and near-linear behavior of the phases with no frequency mixing to higher harmonics through the nonlinear terms. Clearly, these are severe assumptions which limit the validity of the resulting predictions, but, as we shall see, we find fairly close agreement between our perturbation analysis and full numerical simulations of the phase equations.

The above ansatz reads for the equivalent system, Eqs. (7) and (8),

$$
\begin{aligned}
u= & u_{0}+\Gamma x+\frac{1+\sigma}{2} \omega t+\sum_{m=0}^{\infty} \\
& \times\left(\frac{A_{m}+C_{m}}{2} \cos \omega t+\frac{B_{m}+D_{m}}{2} \sin \omega t\right) \cos k_{m} x, \\
v= & v_{0}+\frac{1-\sigma}{2} \omega t+\sum_{m=0}^{\infty} \\
& \times\left(\frac{A_{m}-C_{m}}{2} \cos \omega t+\frac{B_{m}-D_{m}}{2} \sin \omega t\right) \cos k_{m} x,
\end{aligned}
$$

where $u_{0}=\left(\phi_{0}+\psi_{0}\right) / 2$ and $v_{0}=\left(\phi_{0}-\psi_{0}\right) / 2$. Inserting these into Eqs. (7) and (8), multiplying the equations by $\cos k_{n} x$, 
integrating the spatial dimension, and maintaining only terms of up to first order in $A_{n}, B_{n}, C_{n}$, and $D_{n}$, yields for terms oscillating at $\omega$ the following two equations from which $A_{n}, B_{n}, C_{n}$, and $D_{n}$ can be extracted:

$$
\begin{gathered}
{\left[\left(1-\Delta_{2}\right) \omega^{2}-\left(1+\Delta_{1}\right) k_{n}^{2}\right]\left[\frac{A_{n}+C_{n}}{2} \cos \omega t+\frac{B_{n}+D_{n}}{2} \sin \omega t\right]+\alpha \omega\left[\frac{A_{n}+C_{n}}{2} \sin \omega t-\frac{B_{n}+D_{n}}{2} \cos \omega t\right]} \\
=\cos \left(\frac{1-\sigma}{2} \omega t+v_{0}\right)\left[Z_{n}^{(2)}(\Gamma L) \cos \left(\frac{1+\sigma}{2} \omega t+u_{0}\right)-Z_{n}^{(1)}(\Gamma L) \sin \left(\frac{1+\sigma}{2} \omega t+u_{0}\right)\right], \\
{\left[\left(1+\Delta_{2}\right) \omega^{2}-\left(1-\Delta_{1}\right) k_{n}^{2}\right]\left[\frac{A_{n}-C_{n}}{2} \cos \omega t+\frac{B_{n}-D_{n}}{2} \sin \omega t\right]+\alpha \omega\left[\frac{A_{n}-C_{n}}{2} \sin \omega t-\frac{B_{n}-D_{n}}{2} \cos \omega t\right]} \\
=\sin \left(\frac{1-\sigma}{2} \omega t+v_{0}\right)\left[Z_{n}^{(1)}(\Gamma L) \cos \left(\frac{1+\sigma}{2} \omega t+u_{0}\right)+Z_{n}^{(2)}(\Gamma L) \sin \left(\frac{1+\sigma}{2} \omega t+u_{0}\right)\right],
\end{gathered}
$$

where $Z_{n}^{(1)}(\Gamma L)$ and $Z_{n}^{(2)}(\Gamma L)$ are given by

$$
\begin{aligned}
Z_{n}^{(1)}(\Gamma L) & =\frac{2}{L\left(1+\delta_{n, 0}\right)} \int_{0}^{L} \cos \Gamma x \cos k_{n} x d x \\
& =-\frac{2 \Gamma L}{1+\delta_{n, 0}} \frac{(-1)^{n} \sin \Gamma L}{(n \pi)^{2}-(\Gamma L)^{2}}, \\
Z_{n}^{(2)}(\Gamma L) & =\frac{2}{L\left(1+\delta_{n, 0}\right)} \int_{0}^{L} \cos \Gamma x \sin k_{n} x d x \\
& =-\frac{2 \Gamma L}{1+\delta_{n, 0}} \frac{1-(-1)^{n} \cos \Gamma L}{(n \pi)^{2}-(\Gamma L)^{2}},
\end{aligned}
$$

and where $\delta_{m, n}=1$ for $m=n$ and $\delta_{m, n}=0$ for $m \neq n$. From Eqs. (17) and (18) can then be extracted the coefficients, $A_{n}, B_{n}, C_{n}$, and $D_{n}$. Note that unlike the case of kink solitons, where good trial functions can be generated only for the extreme cases of $\phi=\psi$ and $\phi=-\psi$ (see, e.g., Ref. 9), we can here find a useful approximate solution for all phase differences between the two phases. This is essential when finding the locking range at a given frequency, since this range is determined by the dynamics of the modes between the two extreme cases, $\phi=\psi$ and $\phi=-\psi$.

In the following we will, for simplicity, consider the cases of unidirectional $(\sigma=1)$ and antidirectional $(\sigma=-1)$ propagation separately.

\section{A. $\boldsymbol{\sigma} \equiv \mathbf{1}$}

This case implies that the normalized voltage drops $\left(\omega_{1}\right.$ and $\left.\omega_{2}\right)$,

$$
\begin{aligned}
& \omega_{1}=\frac{1}{L}\left\langle\int_{0}^{L} \phi_{t} d x\right\rangle, \\
& \omega_{2}=\frac{1}{L}\left\langle\int_{0}^{L} \psi_{t} d x\right\rangle,
\end{aligned}
$$

of the two systems must be identical. Since there is no ac drive on the system we can choose to omit one of the constant phases. In this case we choose to eliminate $u_{0}$ from the calculations, since $v_{0}$ holds the time difference between the locked Fiske modes. We then find the amplitudes of the induced resonances from Eqs. (17) and (18) to be

$$
\begin{gathered}
\frac{A_{n}+C_{n}}{2}=\frac{Z_{n}^{(2)}(\Gamma L)\left[\left(1-\Delta_{2}\right) \omega^{2}-\left(1+\Delta_{1}\right) k_{n}^{2}\right]+Z_{n}^{(1)}(\Gamma L) \alpha \omega}{\left[\left(1-\Delta_{2}\right) \omega^{2}-\left(1+\Delta_{1}\right) k_{n}^{2}\right]^{2}+(\alpha \omega)^{2}} \cos v_{0}, \\
\frac{B_{n}+D_{n}}{2}=\frac{Z_{n}^{(1)}(\Gamma L)\left[\left(1-\Delta_{2}\right) \omega^{2}-\left(1+\Delta_{1}\right) k_{n}^{2}\right]-Z_{n}^{(2)}(\Gamma L) \alpha \omega}{\left[\left(1-\Delta_{2}\right) \omega^{2}-\left(1+\Delta_{1}\right) k_{n}^{2}\right]^{2}+(\alpha \omega)^{2}} \cos v_{0}, \\
\frac{A_{n}-C_{n}}{2}=\frac{Z_{n}^{(1)}(\Gamma L)\left[\left(1+\Delta_{2}\right) \omega^{2}-\left(1-\Delta_{1}\right) k_{n}^{2}\right]-Z_{n}^{(2)}(\Gamma L) \alpha \omega}{\left[\left(1+\Delta_{2}\right) \omega^{2}-\left(1-\Delta_{1}\right) k_{n}^{2}\right]^{2}+(\alpha \omega)^{2}} \sin v_{0}, \\
\frac{B_{n}-D_{n}}{2}=\frac{-Z_{n}^{(2)}(\Gamma L)\left[\left(1+\Delta_{2}\right) \omega^{2}-\left(1-\Delta_{1}\right) k_{n}^{2}\right]-Z_{n}^{(1)}(\Gamma L) \alpha \omega}{\left[\left(1+\Delta_{2}\right) \omega^{2}-\left(1-\Delta_{1}\right) k_{n}^{2}\right]^{2}+(\alpha \omega)^{2}} \sin v_{0} .
\end{gathered}
$$


The corresponding dc component of the dynamics is then found by inserting Eqs. (15), (16), and (21)-(24) into Eq. (7). We are then able to evaluate the normalized dc currentvoltage $\left[\eta_{s}-\omega(\mathrm{I}-\mathrm{V})\right]$ characteristics of the coupled and locked system as

$$
\begin{aligned}
\eta_{s}= & \alpha \omega+\frac{1}{2} \sum_{n=-\infty}^{\infty} \frac{(\Gamma L / 2)^{2} \sin ^{2}[(\Gamma L-n \pi) / 2]}{[(\Gamma L+n \pi) / 2]^{2}[(\Gamma L-n \pi) / 2]^{2}} \\
& \times\left[\frac{\alpha \omega \sin ^{2} v_{0}}{\left[\left(1+\Delta_{2}\right) \omega^{2}-\left(1-\Delta_{1}\right) k_{n}^{2}\right]^{2}+(\alpha \omega)^{2}}\right. \\
& \left.+\frac{\alpha \omega \cos ^{2} v_{0}}{\left[\left(1-\Delta_{2}\right) \omega^{2}-\left(1+\Delta_{1}\right) k_{n}^{2}\right]^{2}+(\alpha \omega)^{2}}\right] .
\end{aligned}
$$

This expression is valid when the second term on the righthand side is relatively small, i.e., when the locked Fiske steps are small or are on the parts of the I-V curve close to the Ohmic line. The internal phase, $v_{0}$, determines if the mode consists of two identical Fiske modes $\left(v_{0}=0\right)$, two opposite $\left(v_{0}=\pi / 2\right)$, or a mixture of the two extreme cases. As can be seen from Eq. (25) the two extreme cases have their resonances at different frequencies, which is equivalent to the split velocities of kink solitons discussed in Refs. 7-9. The internal phase can be controlled by the external bias currents, since the difference in bias currents can be shown to have the following relationship with the internal phase [this is done by inserting Eqs. (15), (16), and (21)-(24) into Eq. (8)]:

$$
\begin{aligned}
\eta_{d}= & \sin \left(2 v_{0}\right) \frac{1}{4} \sum_{n=-\infty}^{\infty} \frac{(\Gamma L / 2)^{2} \sin ^{2}[(\Gamma L-n \pi) / 2]}{[(\Gamma L+n \pi) / 2]^{2}[(\Gamma L-n \pi) / 2]^{2}} \\
& \times\left[\frac{\left(1+\Delta_{2}\right) \omega^{2}-\left(1-\Delta_{1}\right) k_{n}^{2}}{\left[\left(1+\Delta_{2}\right) \omega^{2}-\left(1-\Delta_{1}\right) k_{n}^{2}\right]^{2}+(\alpha \omega)^{2}}\right. \\
& \left.-\frac{\left(1-\Delta_{2}\right) \omega^{2}-\left(1+\Delta_{1}\right) k_{n}^{2}}{\left[\left(1-\Delta_{2}\right) \omega^{2}-\left(1+\Delta_{1}\right) k_{n}^{2}\right]^{2}+(\alpha \omega)^{2}}\right] .
\end{aligned}
$$

This relationship also gives the limits to the difference in dc bias for which the locked states of Fiske modes can exist. Varying $v_{0}$ from $-\pi / 4$ to $\pi / 4$ requires the difference in bias, $\eta_{d}$, to vary between its extreme values which can easily be obtained from Eq. (26).

\section{B. $\sigma \equiv-1$}

When the two systems are biased with forces of opposite signs, the two phases, $\phi$ and $\psi$, evolve in opposite directions as well $\left(\omega_{1}=-\omega_{2}\right)$. In this case we can therefore choose to omit $v_{0}$ from the calculations since this phase does not contain important information about the locked state. We then find the counter pairs of Eqs. (21)-(24) for this case:

$$
\begin{aligned}
\frac{A_{n}+C_{n}}{2}= & {\left[Z_{n}^{(2)}(\Gamma L) \cos u_{0}+Z_{n}^{(1)}(\Gamma L) \sin u_{0}\right] } \\
& \times \frac{\left(1-\Delta_{2}\right) \omega^{2}-\left(1+\Delta_{1}\right) k_{n}^{2}}{\left[\left(1-\Delta_{2}\right) \omega^{2}-\left(1+\Delta_{1}\right) k_{n}^{2}\right]^{2}+(\alpha \omega)^{2}},
\end{aligned}
$$

$$
\begin{aligned}
\frac{B_{n}+D_{n}}{2}= & -\left[Z_{n}^{(2)}(\Gamma L) \cos u_{0}+Z_{n}^{(1)}(\Gamma L) \sin u_{0}\right] \\
& \times \frac{\alpha \omega}{\left[\left(1-\Delta_{2}\right) \omega^{2}-\left(1+\Delta_{1}\right) k_{n}^{2}\right]^{2}+(\alpha \omega)^{2}},
\end{aligned}
$$

$$
\frac{A_{n}-C_{n}}{2}=\left[Z_{n}^{(1)}(\Gamma L) \cos u_{0}-Z_{n}^{(2)}(\Gamma L) \sin u_{0}\right]
$$

$$
\times \frac{\alpha \omega}{\left[\left(1+\Delta_{2}\right) \omega^{2}-\left(1-\Delta_{1}\right) k_{n}^{2}\right]^{2}+(\alpha \omega)^{2}},
$$

$$
\begin{aligned}
\frac{B_{n}-D_{n}}{2}= & {\left[Z_{n}^{(1)}(\Gamma L) \cos u_{0}-Z_{n}^{(2)}(\Gamma L) \sin u_{0}\right] } \\
& \times \frac{\left(1+\Delta_{2}\right) \omega^{2}-\left(1-\Delta_{1}\right) k_{n}^{2}}{\left[\left(1+\Delta_{2}\right) \omega^{2}-\left(1-\Delta_{1}\right) k_{n}^{2}\right]^{2}+(\alpha \omega)^{2}} .
\end{aligned}
$$

As before the dc component of the dynamics can be found by inserting Eqs. (15), (16), and (27)-(30) into the same equations, (7) and (8). The difference in bias, $\eta_{d}$, is now the parameter giving the structure of the $\mathrm{dc}$ behavior, and the sum of the biases, $\eta_{s}$, gives the range of locking as well as the constant phase, $u_{0}$,

$$
\begin{aligned}
\eta_{d}= & \alpha \omega+\frac{1}{2} \sum_{n=-\infty}^{\infty} \frac{(\Gamma L / 2)^{2} \sin ^{2}[(\Gamma L-n \pi) / 2]}{[(\Gamma L+n \pi) / 2]^{2}[(\Gamma L-n \pi) / 2]^{2}} \\
& \times\left[\frac{\alpha \omega \sin ^{2}\left[\widetilde{u_{0}}+n(\pi / 2)\right]}{\left[\left(1+\Delta_{2}\right) \omega^{2}-\left(1-\Delta_{1}\right) k_{n}^{2}\right]^{2}+(\alpha \omega)^{2}}\right. \\
+ & \left.\frac{\alpha \omega \cos ^{2}\left[\widetilde{u_{0}}+n(\pi / 2)\right]}{\left[\left(1-\Delta_{2}\right) \omega^{2}-\left(1+\Delta_{1}\right) k_{n}^{2}\right]^{2}+(\alpha \omega)^{2}}\right], \\
\eta_{s}= & \sin \left(2 \widetilde{u_{0}}\right) \frac{1}{4} \sum_{n=-\infty}^{\infty}(-1)^{n} \\
& \times \frac{(\Gamma L / 2)^{2} \sin ^{2}[(\Gamma L-n \pi) / 2]}{[(\Gamma L+n \pi) / 2]^{2}[(\Gamma L-n \pi) / 2]^{2}} \\
& \times\left[\frac{\left(1+\Delta_{2}\right) \omega^{2}-\left(1-\Delta_{1}\right) k_{n}^{2}}{\left[\left(1+\Delta_{2}\right) \omega^{2}-\left(1-\Delta_{1}\right) k_{n}^{2}\right]^{2}+(\alpha \omega)^{2}}\right. \\
& \left.-\frac{\left(1-\Delta_{2}\right) \omega^{2}-\left(1+\Delta_{1}\right) k_{n}^{2}}{\left[\left(1-\Delta_{2}\right) \omega^{2}-\left(1+\Delta_{1}\right) k_{n}^{2}\right]^{2}+(\alpha \omega)^{2}}\right],
\end{aligned}
$$


where $\widetilde{u_{0}}=u_{0}+\Gamma L / 2$. As in the above case, we can extract the locking range by varying $\widetilde{u_{0}}$ between $-\pi / 4$ and $\pi / 4$ and observe the extreme values of Eq. (32).

It is important to notice that both pairs of expressions Eqs. (25) and (26) and (31) and (32) are, under the given approximations, valid for all Fiske modes (all $n$ ), including the socalled flux-flow region, where $|\omega| \approx k_{n} \approx|\Gamma|$. It is also worth noting that the above expressions predict the situation where an unbiased system can be "pulled" into frequency locking by its biased neighbor operated in a Fiske mode. This situation is found for $\left|\eta_{s}\right|=\left|\eta_{d}\right|$. It is further interesting that the above expressions Eqs. (31) and (32) also predict that an unbiased system $\left(\left|\eta_{s}\right|=\left|\eta_{d}\right|\right)$ can be driven into a mode where

$$
\left\langle\int_{0}^{L} \phi_{t} d x\right\rangle=-\left\langle\int_{0}^{L} \psi_{t} d x\right\rangle
$$

\section{NUMERICAL SIMULATIONS}

We have performed direct numerical simulations of the coupled phase equations using an explicit second-order finite difference Verlet-type integrator in time and space for the system given by Eqs. (7) and (8). The spatial and temporal discretizations, $d t \leqslant d x$, were varied up to $d x=0.05$ in order to ensure sufficient resolution of the modes in time and space. For a single sine-Gordon system, stability of the Verlet integrator requires $d t \leqslant d x$, while [due to the characteristic velocity, $\left.u=\sqrt{\left(1+\Delta_{1}\right) /\left(1-\Delta_{2}\right)}\right]$ the coupled system requires $d t<d x$. All simulations were performed by allowing a transient time of the dynamics to be at least 4000 normalized time units followed by an equal averaging time for each acquired number. Given the large parameter space, we have fixed the boundary condition at $\Gamma=9.5$. We have further limited our comparisons to the case of inductive coupling $\Delta_{1}\left(\Delta_{2}=0\right)$. This has been done for two reasons. First, magnetic coupling has been considered the important mechanism for Josephson systems. (In Ref. 8 it was found that the small amplitude limit of the capacitively coupled system is equivalent to coupled nonlinear Schrödinger equations modeling coupled optical fibers. ${ }^{35}$ ) Second, other publications, e.g., Ref. 9, have demonstrated that comparisons between perturbation analyses and numerical simulations for the capacitive coupling behave with the same reliability as for the inductive coupling.

The quantities we have decided to compare are the locking ranges between the two systems with nonequal biases and we therefore started the systems in a given Fiske mode with equal $\left(\left|\eta_{1}\right|=\left|\eta_{2}\right|\right)$ bias. We then increased the difference between $\left|\eta_{1}\right|$ and $\left|\eta_{2}\right|$ in small increments, while keeping the average of $\left|\eta_{1}\right|$ and $\left|\eta_{2}\right|$ constant. The individual average frequencies were monitored during this procedure to determine if the coupled systems were phase locked or not.

In Fig. 1 we have shown the $I-V$ curves for a system with parameters $L=2, \alpha=0.1$, and $\Delta_{1}=0.1$. The curves are shown near the flux-flow modes, which in this case are the Fiske modes centered around $n=6$. Figure 1(a) shows the $I$ - $V$ curves for $\eta_{s}=\eta_{1}=\eta_{2}(\sigma=1)$ with solid curves representing the expressions Eq. (26) for $v_{0}=0$ and $v_{0}=\pi / 2$. The dots (-) represent the results of the numerical simulations. The mode indicated with $\mathrm{FS} n^{(+)}$is the $n$th Fiske step where $\phi=\psi\left(v_{0}=0\right)$ and the one indicated by $\mathrm{FSn}^{(-)}$is the $v_{0}=\pi / 2$ mode where $\phi_{x} \approx-\psi_{x}$. Thus, as can be seen from Eqs. (25) and (31), the usual Fiske resonance at $\omega=k_{n}$ is now split into two resonances at $\omega=\sqrt{\left(1 \pm \Delta_{1}\right) /\left(1 \mp \Delta_{2}\right)} k_{n}$. Note that only for the $v_{0}=0$, $\sigma=1$, and $\eta_{s}=0$ modes can we rescale the Fiske steps to the uncoupled ones described in Ref. 33. Due to the boundary conditions a direct scaling cannot be made in any other case. Figure 1(a) illustrates the good comparison between the predicted split Fiske resonances and the numerical results when the two systems are biased equally. As the difference in bias is increased, while the sum is kept constant, the dynamics changes since the internal phase $v_{0}$ is determined by $\eta_{d}$ [see Eq. (26)]. Figure 1(b) shows the $I-V$ curve for the extreme value of $\eta_{d}$, where the two Fiske modes unlock and $\left|v_{0}\right|=\pi / 4$, while Fig. 1(c) shows the maximum value of $\eta_{d}$ for which the system stays in a locked state of Fiske modes. Here, the solid curve represents the maximum value of expression Eq. (26) (for $v_{0}=\pi / 4$ ) and the dots represent numerical simulations. The dots are displayed as pairs, where the lower is a value of $\eta_{d}$ in a locked state and the upper is a value of an unlocked state. The agreement between our analysis [see Eqs. (25) and (26)] and the numerical results is very good, as is obvious from Fig. 1(c).

In Fig. 2 we have shown the same comparison for the $\sigma=-1$ modes. In this case we keep $\eta_{d}$ constant and vary $\eta_{s}$ from 0 until the system unlocks. We find almost the same locking range, and the simulations are in as good agreement with the prediction as in the case of $\sigma=1$ shown in Fig. 1. Using the fluxon picture for the flux-flow mode, this means that phase-locking between two fluxon arrays is achieved as easily when they move in the opposite direction as when they move in the same direction-and the two locking phenomena are described by the same mechanism. In Fig. 3 are shown examples of comparisons in the parts of the parameter space where the first Fiske steps $\left(\mathrm{FS}^{( \pm)}\right)$are localized. We have here chosen to display the comparison in locking-range only. Figure 3(a) shows the case $\sigma=1$, and Fig. 3(b) shows $\sigma=-1$. Reasonably good agreement between analysis and simulations is again apparent, although the $\mathrm{FS} 1^{(+)}$modes have weaker locking than predicted. Figures 3(c) and 3(d) show the same system, but for $\Delta_{1}=0.2$. Here we find better agreement than in Figs. 3(a) and 3(b). The reason for this is, of course, the relatively primitive analysis given in Sec. II. When $\Delta_{1}$ is small, $\mathrm{FS}^{(+)}$and $\mathrm{FS} n^{(-)}$are closely positioned on the frequency scale. Depending on the dissipation parameter, the two resonances will mix through the nonlinearity of the system and deviations from the single-mode analysis given above may be expected. For moderately large coupling constants this becomes less of a problem as the splitting between the characteristic velocities becomes larger. However, if the coupling is large enough for, e.g., $\mathrm{FS}^{(-)}$to be close to $\mathrm{FS}^{(+)}$, this problem arises again. These are therefore important considerations when designing coupled Josephson systems with optimized locking capabilities.

Yet another important observation is that the locking range seems to saturate with increasing coupling. The locking range for $\Delta_{1}=0.2$ is of the same magnitude as for $\Delta_{1}=0.1$. It is therefore not necessarily desirable with respect 

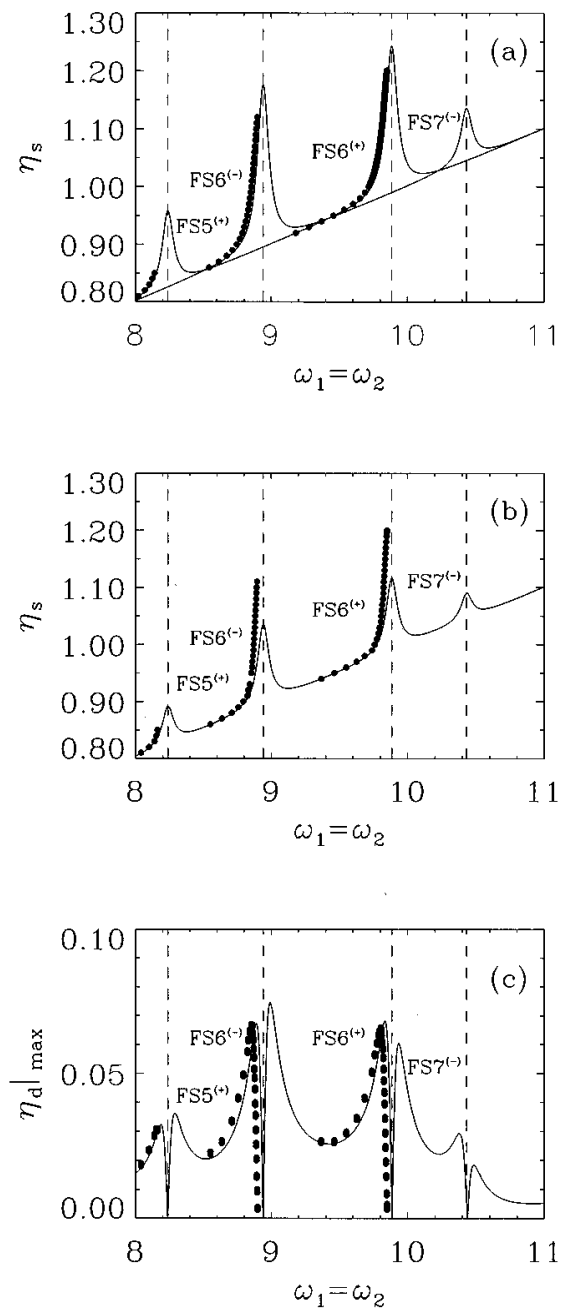

FIG. 1. Normalized current-voltage characteristics and locking ranges for the system given by Eqs. (1)-(4) with parameters: $L=2, \Gamma=9.5, \alpha=0.1, \Delta_{1}=0.1, \Delta_{2}=0$, and $\sigma=1$. Lines represent the result of the analysis, Eqs. (25) and (26), and shows the result of numerical simulations. The Fiske resonances are shown as vertical dashed lines. (a) $\eta_{s}$ vs $\omega$; $\eta_{d}=0, v_{0}=0$. (b) $\eta_{s}$ vs $\omega$; Maximum $\eta_{d}$ and $v_{0}=\pi / 4$. (c) $\eta_{d}$ vs $\omega$; The locking range is given by the maximum value of Eq. (26) for $v_{0}=\pi / 4$.

to phase locking to simply increase the coupling between the two systems. This is in agreement with the results for fluxon locking, ${ }^{7,9}$ where a moderate coupling parameter $\left(\Delta_{1} \approx 0.1\right)$ in many cases creates roughly the same locking range as a larger coupling parameter.

In order to demonstrate the validity of the present analysis for longer systems, we have performed simulations for $L=10$ as well. Figures 4 and 5 summarize the simulations for the first Fiske step $\left(\mathrm{FS} 1^{( \pm)}\right)$for a system given by $\alpha=0.1$ and $\Delta_{1}=0.1$. The comparisons for the $I-V$ curves for $v_{0}=0$ (solid) and $v_{0}=\pi / 2$ (dashed) show reasonable, but not perfect, agreement. In both Figs. 4(a) $(\sigma=1)$ and 5(a) $(\sigma=-1)$ we observe a smaller deviation from the linear curves than predicted by the theory. This is in good agreement with the assumptions built into the analysis. The corresponding locking ranges between the Fiske modes are shown in Figs. 4(b) and 5(b). As for the $L=2$ cases we find relatively good agreement between analysis and simulations. A
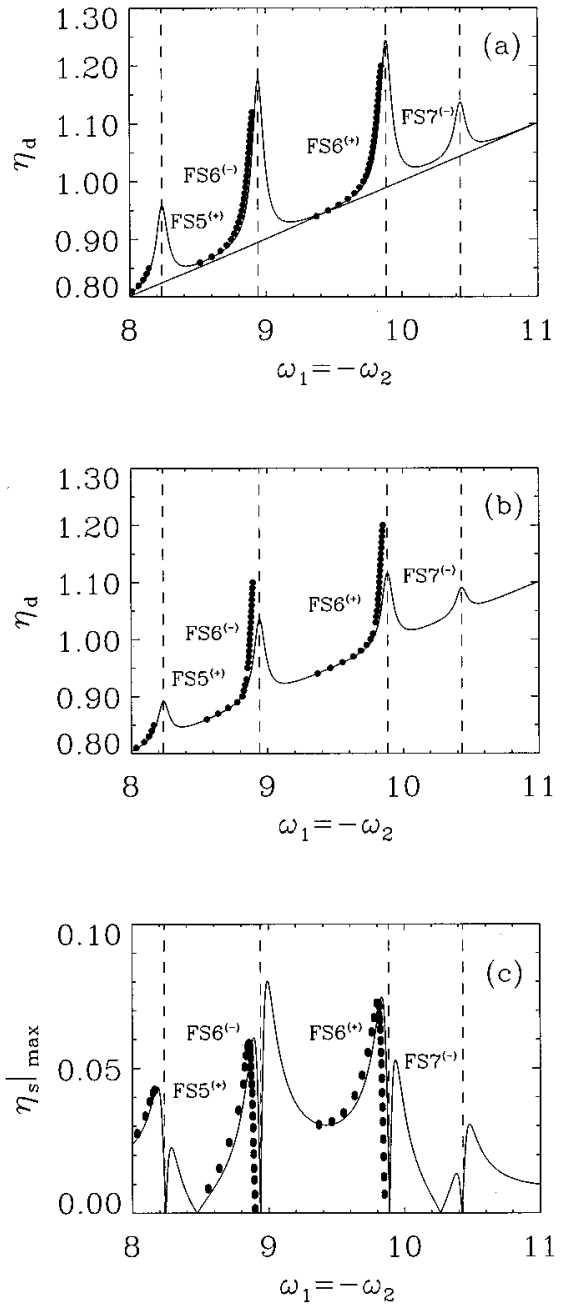

FIG. 2. Normalized current-voltage characteristics and locking ranges for the system given by Eqs. (1)-(4) with parameters: $L=2, \Gamma=9.5, \alpha=0.1, \Delta_{1}=0.1, \Delta_{2}=0$, and $\sigma=-1$. Lines represent the result of the analysis, Eqs. (31) and (32), and shows the result of numerical simulations. The Fiske resonances are shown as vertical dashed lines. (a) $\eta_{d}$ vs $\omega ; \eta_{s}=0, \widetilde{u_{0}}=0$. (b) $\eta_{d}$ vs $\omega$; Maximum $\eta_{s}$ and $\widetilde{u_{0}}=\pi / 4$. (c) $\eta_{s}$ vs $\omega$; The locking range is given by the maximum value of Eq. (32) for $\widetilde{u_{0}}=\pi / 4$.

general trend seems to be that the "fast" modes $\left(\mathrm{FS} 1^{(+)}\right)$ have smaller locking ranges than predicted, whereas the slow modes $\left(\mathrm{FS}^{(-)}\right)$are given almost perfectly by the expressions above. Still, we note that all qualitative features of the locking range and the order of magnitude seem to be well addressed by the above description of the dynamics. It should be pointed out that the analysis in this paper contains no information about the stability of the observed modesonly about their existence. We can therefore not give general comments on when to expect the different branches on the $I-V$ curves to be relevant. A comprehensive stability analysis was given in Ref. 9 for isolated kink solitons in coupled systems. This analysis, using the profile of the soliton, led to the conclusion that the fast (slow) mode of kink solitons could never (only) be stable at velocities $u<\sqrt{1-\Delta_{1}} / \sqrt{1+\Delta_{2}}$. Given the close relation between kink solitons and Fiske modes, it might be expected that the same would be true for Fiske states. However, looking at Fig. 4(a), 

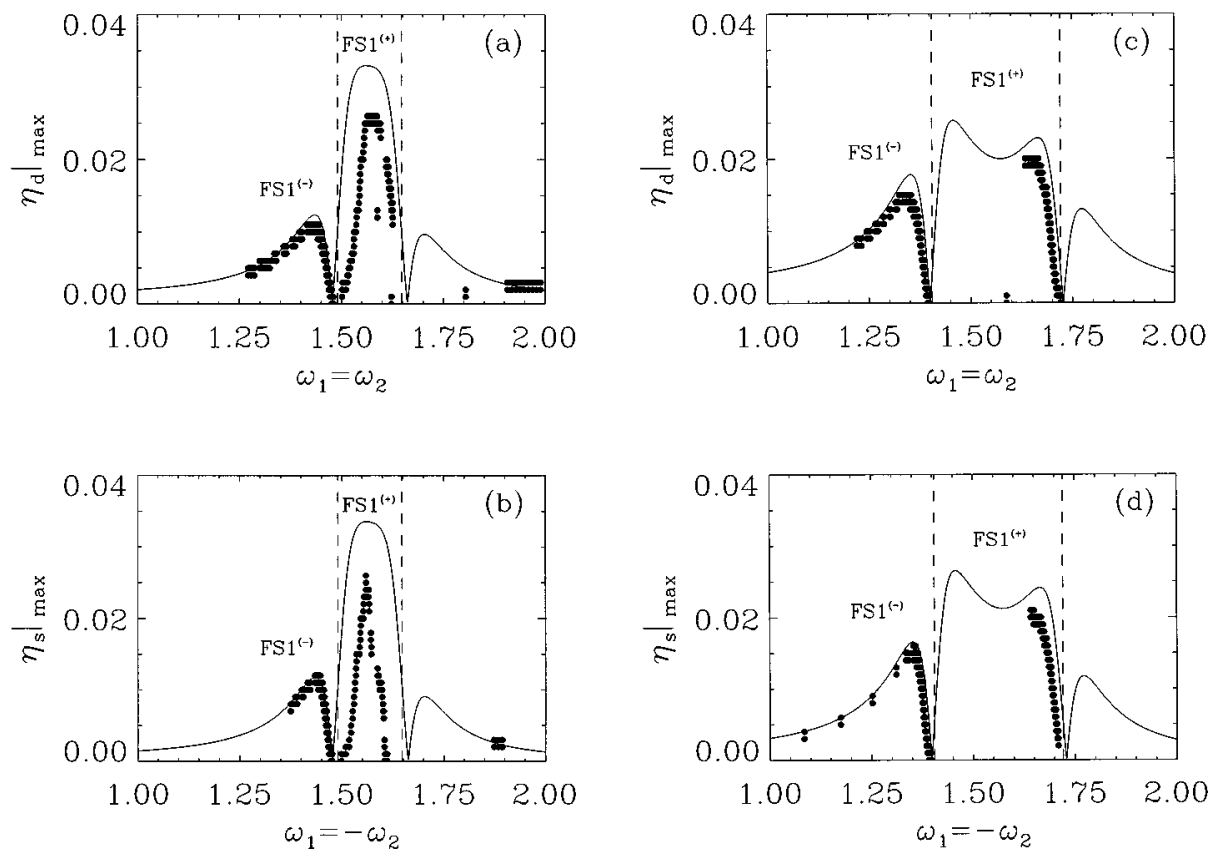

FIG. 3. The locking ranges in bias for a system given by $L=2, \Gamma=9.5, \alpha=0.1$, and $\Delta_{2}=0$. The Fiske resonances are shown as vertical dashed lines. Dots $(-)$ represent the results of numerical simulations of Eqs. (1)-(4) and solid lines represent the results of the analysis, Eq. (26) $\left(\sigma=1\right.$ and $\left.\Delta_{1}=0.1\right)$ (a), Eq. (32) $\left(\sigma=-1\right.$ and $\left.\Delta_{1}=0.1\right)$ (b), Eq. (26) $\left(\sigma=1\right.$ and $\left.\Delta_{1}=0.2\right)\left(\right.$ c), Eq. (32) $\left(\sigma=-1\right.$ and $\left.\Delta_{1}=0.2\right)($ d).
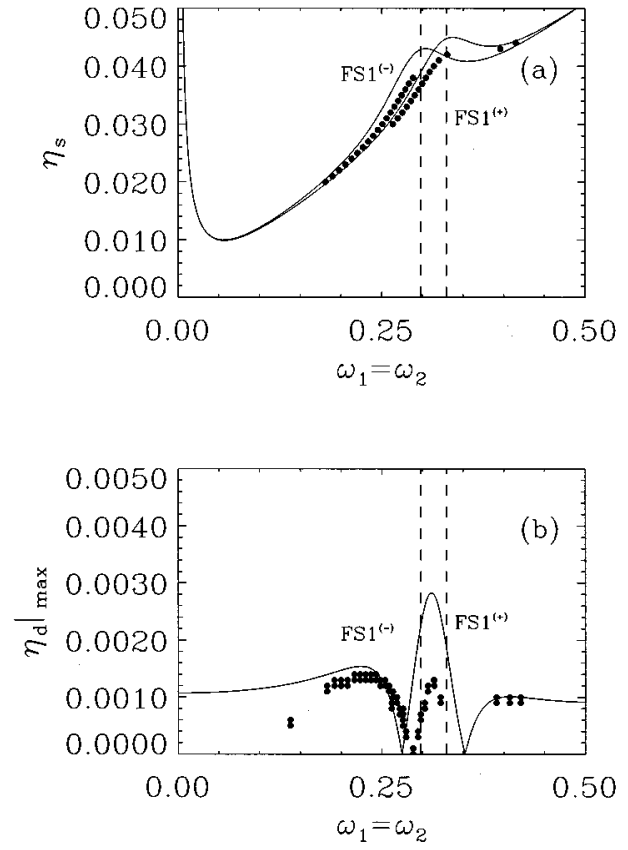

FIG. 4. Normalized current-voltage characteristics and locking ranges for the system given by Eqs. (1)-(4) with parameters: $L=10, \Gamma=9.5, \alpha=0.1, \Delta_{1}=0.1, \Delta_{2}=0$, and $\sigma=1$. Lines represent the result of the analysis, Eqs. (25) and (26), and shows the result of numerical simulations. The Fiske resonances are shown as vertical dashed lines. (a) $\eta_{s}$ vs $\omega ; \eta_{d}=0, v_{0}=0$. (b) $\eta_{d}$ vs $\omega$; The locking range is given by the maximum value of Eq. (26) for $v_{0}=\pi / 4$.
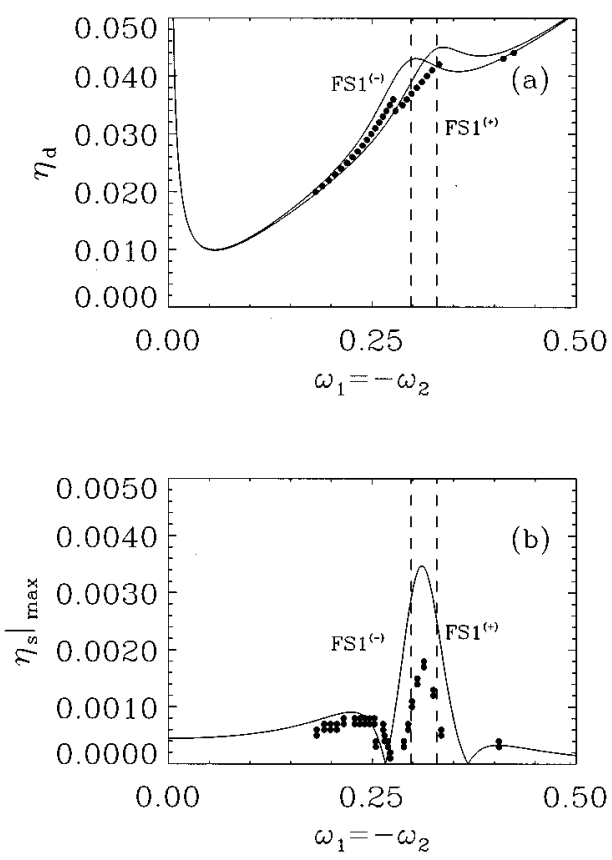

FIG. 5. Normalized current-voltage characteristics and locking ranges for the system given by Eqs. (1)-(4) with parameters: $L=10, \Gamma=9.5, \alpha=0.1, \Delta_{1}=0.1, \Delta_{2}=0$, and $\sigma=-1$. Lines represent the result of the analysis, Eqs. (31) and (32), and shows the result of numerical simulations. The Fiske resonances are shown as vertical dashed lines. (a) $\eta_{d}$ vs $\omega ; \eta_{s}=0, \tilde{u_{0}}=0$. (b) $\eta_{s}$ vs $\omega$; The locking range is given by the maximum value of Eq. (32) for $\tilde{u_{0}}=\pi / 4$. 
we find a large overlap in frequency (speed) between the two branches of FS1. Thus, the stability information from kink solitons cannot be extrapolated over to the Fiske and fluxflow modes discussed here. The analysis predicts the locking range to be independent of which branch (fast or slow) the dynamics is operated at; i.e., only the frequency determines the locking range. Figure 4 also demonstrates this feature. Indeed, for the frequency range of overlap between $\mathrm{FS}^{(+)}$ and $\mathrm{FS}^{(-)}$in Fig. 4(a), we find the corresponding locking ranges in Fig. 4(b) to be independent of which branch is considered [Fig. 4(b) has two sets of overlapping dots, representing the phase-locking simulations of the two modes].

Finally, comparisons for the $L=10$ case in the flux-flow region are shown. The dynamical behavior turned out to be extremely complicated in this case and we found mostly chaotic dynamics for $\alpha=0.1$ when operating the system near $|\omega|=|\Gamma|$. This resulted in inherently unstable resonances which have therefore been omitted in this the presentation. The reason for the complicated behavior is a combination of proximity to the strong flux-flow resonance, where $|\omega| \approx \Gamma$, and the length of the system. In fact, the system length determines the frequency spacing between the fundamental Fiske resonances, $\delta \omega_{L}=\pi / L$, whereas the coupling determines the spacing between the split modes, $\delta \omega_{\Delta_{1}}$ $=\sqrt{1+\Delta_{1}}-\sqrt{1-\Delta_{1}}$. Thus, a long system will create more interference and mixing between the split modes of different Fiske resonances. Figure 6 indicates all the split Fiske resonances in the flux-flow region. The indicated frequency region includes (dotted) $\mathrm{FS} n^{(-)}$with $n=27-36$ and (dashed) $\mathrm{FS} n^{(+)}$with $n=25-33$. The potential for very complicated frequency mixing between various Fiske modes is clearly seen. We have therefore damped the dynamics by increasing the dissipation parameter to $\alpha=0.2$. Figure 6 shows comparisons between the analysis and simulations for the locking range in a system with $L=10, \alpha=0.2$, and $\Delta_{1}=0.1$. The results span several of the resonances, and excellent agreement is found between the analysis and the simulations for both $\sigma=1$ [Fig. 6(a)] and $\sigma=-1$ [Fig. 6(b)]; even for this very complicated case. The most significant difference between the simulated locking ranges and the analysis is that a slight frequency shift seems to be present in the figures. This is, however, a minor detail, which is not surprising given the severe approximations built into the analysis.

\section{DISCUSSION}

We have analyzed a system of two inductively and capacitively coupled sine-Gordon phases, which in the inductive case has been used as a model for magnetically coupled long Josephson junctions. Experimentally, the most frequently studied Fiske and flux-flow resonances, ${ }^{10-12,25,26}$ occur when an external dc magnetic field is applied to the system in order to force flux penetration through the boundaries of the junctions. The synchronized states have been observed experimentally and even the $\sigma=-1$ case, where the voltages of two Josephson junctions have opposite signs, have recently been observed experimentally in Ref. 12 . We have developed a spatial multimode theory for the interaction of two equal resonances and evaluated the range in bias difference for which these modes stay locked at the same fre-
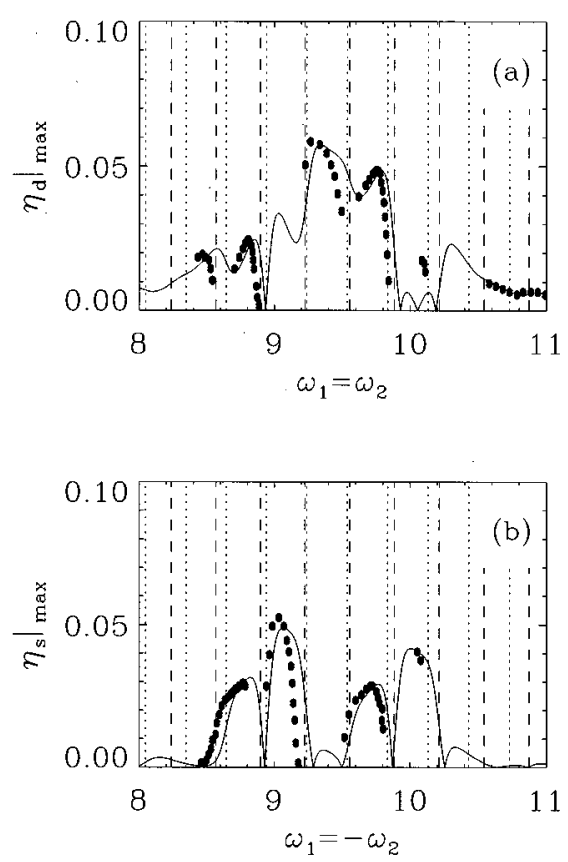

FIG. 6. The locking ranges in bias for a system given by $L=10, \Gamma=9.5, \alpha=0.2, \Delta_{1}=0.1$, and $\Delta_{2}=0$. The Fiske resonances $\mathrm{FS} n^{(-)}$, for $n=27-36$ are shown as vertical dotted lines and $\mathrm{FS} n^{(+)}$, for $n=25-33$ are shown as vertical dashed lines. Dots (O) represent the results of numerical simulations of Eqs. (1)-(4) and solid lines represent the results of the analysis, Eq. (26) $(\sigma=1)(\mathrm{a})$, and Eq. (32) $(\sigma=-1)(\mathrm{b})$.

quency. Very good agreement is found between the analysis and numerical simulations of both short and long systems for both the flux-flow and the low-order Fiske steps. Apart from describing the coupled modes, and hereby understanding phase locking between the various Fiske modes of equal and opposite bias and voltage, we have noted several useful features for experimental systems. One of the most important observations is that phase locking seems to saturate with moderate coupling, as was also found for phase locking between fluxon modes. The reason for the saturation seems to be the interference between the various split Fiske resonances in the coupled system. This interference gets worse for longer systems since the frequency spacing between the average of the Fiske resonances decreases with length $(L)$. We have also noticed the dynamics becoming complicated as the interference gets stronger. If phase locking between periodic states of Fiske modes is the goal of inductively coupled Josephson junctions, a large coupling parameter is therefore not necessarily the optimal situation. On the other hand, we have also observed that a too weak coupling resulted in complicated dynamics and locking ranges smaller than predicted. The reason for this is closely related to the discussion above. For small coupling parameters we only see the frequency mixing between the split resonances of the same basic Fiske mode. Similarly, a shorter system is probably to be preferred over a system of long junctions, since the Fiske resonances then will be well separated. These considerations should also include the damping parameter $\alpha$, since this determines the width of the resonances, and 
thereby the possible mixing. Large damping does stabilize the dynamics, but it also broadens the interaction in frequency. We finally note that a large value of the normalized external magnetic field, $\widetilde{\Gamma}$, seems always to stabilize the dynamics and give a much broader range of predictable behavior.

\section{ACKNOWLEDGMENTS}

Parts of this work were performed under the auspices of the United States Department of Energy. Financial support was provided by the Natural Sciences and Engineering Research Council of Canada.
${ }^{1}$ K. L. Ngai, Phys. Rev. 182, 555 (1969).

${ }^{2}$ M. B. Mineev, G. S. Mkrtchyan, and V. V. Shmidt, J. Low Temp. Phys. 45, 497 (1981).

${ }^{3}$ A. F. Volkov, Pis'ma Zh. Éksp. Teor. Fiz. 45, 299 (1987) [JETP Lett. 45, 376 (1987)]; Prog. High Temp. Conduc. 4, 129 (1987).

${ }^{4}$ T. Holst, J. Bindslev Hansen, N. Grønbech-Jensen, and J. A. Blackburn, Phys. Rev. B 42, 127 (1990).

${ }^{5}$ Niels Grønbech-Jensen and Mogens R. Samuelsen, Phys. Rev. Lett. 74, 170 (1995).

${ }^{6}$ Yu. M. Ivanchenko, Phys. Rev. B 52, 79 (1995).

${ }^{7}$ N. Gronbech-Jensen, P. S. Lomdahl, M. R. Samuelsen, and J. A. Blackburn, Phys. Rev. B 42, 3976 (1990).

${ }^{8}$ Niels Grønbech-Jensen, Ph.D. thesis, The Technical University of Denmark, 1991.

${ }^{9}$ Niels Grønbech-Jensen, David Cai, A. R. Bishop, A. W. C. Lau, and Peter S. Lomdahl, Phys. Rev. B 50, 6352 (1994).

${ }^{10}$ T. Holst, L. E. Guerrero, J. B. Hansen, N. Grønbech-Jensen, and J. A. Blackburn, in Superconducting Devices and Their Applications, edited by H. Koch and H. Lübbig, Springer Proceedings in Physics, Vol. 64 (Springer-Verlag, Berlin, 1992), p. 389.

${ }^{11}$ P. Barbara, A. Ustinov, and G. Costabile, Phys. Lett. A 191, 443 (1994).

${ }^{12}$ P. Barbara, Ph.D. thesis, The Technical University of Denmark, 1995.

${ }^{13}$ Yu. S. Kivshar and B. A. Malomed, Phys. Rev. B 37, 9325 (1988)

${ }^{14}$ T. Holst, J. Bindslev Hansen, N. Gronbech-Jensen, and J. A. Blackburn, IEEE Trans. Mag. 27, 2704 (1991).

${ }^{15}$ Niels Grønbech-Jensen and James A. Blackburn, Phys. Rev. Lett. 70, 1251 (1993); J. Appl. Phys. 74, 6432 (1993).

${ }^{16}$ Niels Grønbech-Jensen, David Cai, and Mogens R. Samuelsen, Phys. Rev. B 48, 16160 (1993).

${ }^{17}$ M. D. Fiske, Rev. Mod. Phys. 36, 221 (1964).

${ }^{18}$ I. O. Kulik, Sov. Phys. Tech. Phys. 12, 111 (1967).

${ }^{19}$ R. E. Eck, D. J. Scalapino, and B. N. Taylor, in Proceedings of the 9th International Conference on Low Temperature Physics, edited by J. G. Daunt et al. (Plenum, New York, 1965); C. S. Owen and D. J. Scalapino, Phys. Rev. 164, 538 (1967).

${ }^{20}$ J. A. Blackburn, J. D. Leslie, and H. J. Smith, J. Appl. Phys. 42, 1047 (1971).

${ }^{21}$ M. P. Sørensen, N. Arley, P. L. Christiansen, R. D. Parmentier, and O. V. Skovgaard, Phys. Rev. Lett. 51, 1919 (1983).

${ }^{22}$ Niels Gronbech-Jensen and Matteo Cirillo, Phys. Rev. B 50, 12851 (1994).

${ }^{23}$ Niels Grønbech-Jensen, Peter S. Lomdahl, and Matteo Cirillo, Phys. Rev. B 51, 11690 (1995).

${ }^{24}$ A. Barone and G. Paternó, Physics and Applications of the Josephson Effect (Wiley, New York, 1982).

${ }^{25}$ A. Ustinov, H. Kohlstedt, M. Cirillo, N. F. Pedersen, and G. Hallmanns, Phys. Rev. B 48, 10614 (1993).

${ }^{26}$ S. Sakai, A. V. Ustinov, H. Kohlstedt, A. Petraglia, and N. F. Pedersen, Phys. Rev. B 50, 12905 (1994).

${ }^{27}$ Y. M. Zhang, E. Carlsson, D. Winkler, G. Brorsson, H. Zirath, and E. Wikborg, IEEE Trans. Appl. Supercond. 5, 3385 (1995).

${ }^{28}$ Hiroya Andoh, Masanobu Kusunoki, Akira Fujimaki, and Hisao Hayakawa, IEEE Trans. Appl. Supercond. 5, 3377 (1995).

${ }^{29}$ A. V. Ustinov, H. Kohlstedt, and C. Heiden, IEEE Trans. Appl. Supercond. 5, 2743 (1995).

${ }^{30}$ A. E. Duwel, H. S. van der Zant, and T. P. Orlando, IEEE Trans. Appl. Supercond. 5, 3357 (1995).

${ }^{31}$ See, e.g., Yuri S. Kivshar and Tatyana K. Soboleva, Physica B 165-166, 1651 (1990).

${ }^{32}$ Niels Grønbech-Jensen, Ole H. Olsen, and Mogens R. Samuelsen, Phys. Lett. A 179, 27 (1993).

${ }^{33}$ I. O. Kulik and I. K. Yanson, Josephson Effect in Superconducting Tunnel Structures (Nauka, Moscow, 1970).

${ }^{34}$ K. Enpuku, K. Yoshida, and F. Irie, J. Appl. Phys. 52, 344 (1981).

${ }^{35}$ F. Kh. Abdulaev, R. M. Abrarov, and S. A. Darmanyan, Opt. Lett. 14, 131 (1989). 